\title{
Vascular flora of Milan Malpensa airport (Lombardy, Italy). Part I: checklist
}

\author{
Marco Martignoni ${ }^{1}$, Enrico Banfi ${ }^{2}$, Gabriele Galasso ${ }^{2 *}$
}

\begin{abstract}
Airport areas have, among flight infrastructures, large green spaces which, not having production purposes, are managed through interventions of minimal disturbance on habitats, with potentially positive effects on the conservation of biodiversity. The checklist of the vascular flora of the airport of Milan Malpensa is here presented. This airport extends for $12.44 \mathrm{~km}^{2}$ in the southwestern portion of the province of Varese (Lombardy, Italy) in the administrative districts of Cardano al Campo, Casorate Sempione, Ferno, Lonate Pozzolo, Samarate, Somma Lombardo and Vizzola Ticino. The collection campaigns were carried out between August 2010 and September 2018. The specimens collected are now kept in the herbarium facility of the Natural History Museum of Milan (MSNM). The floristic list was organized following the arrangement and the nomenclature of the recently published Italian flora checklists. It includes 395 taxa of specific and subspecific rank $(9.35 \%$ of the entire flora of Lombardy) belonging to 247 genera and 72 families. The native taxa are 318 , while the alien ones are 77 . The native/aliens ratio is comparable to the one calculated at the regional level (4.10 vs. 4.42). The endemic contingent includes two taxa: Centaurea nigrescens subsp. pinnatifida, Italian endemic, and Euphrasia cisalpina, endemic to the Alps. The Malpensa airport is still the only site confirmed at the national level for Lepidium heterophyllum and, at the regional level, for Festuca muralis and Euphrasia cisalpina. The ecological characteristics and the data analysis will be discussed in a second contribution.
\end{abstract}

Key words: airoport, Euphrasia cisalpina, Festuca muralis, Lepidium heterophyllum, Malpensa.

Riassunto - Flora vascolare dell'aeroporto di Milano Malpensa (Lombardia, Italia). I parte: checklist.

Le aree aeroportuali presentano, tra le infrastrutture di volo, ampi spazi verdi i quali, non avendo finalità produttive, vengono gestiti mediante interventi di minimo disturbo sull'habitat, con effetti potenzialmente positivi sulla conservazione della biodiversità. Viene qui presentata la checklist della flora vascolare del sedime aeroportuale di Malpensa, esteso per $12,44 \mathrm{~km}^{2}$ nella porzione sud-occidentale della provincia di Varese (Lombardia, Italia) nel territorio dei comuni di Cardano al Campo,

\footnotetext{
${ }^{1}$ Piazza G. Matteotti 25, 21050 Lonate Ceppino (VA), Italia. E-mail: marcomartignoni1974@libero.it

${ }^{2}$ Sezione di Botanica, Museo di Storia Naturale di Milano, Corso Venezia 55, 20121 Milano, Italia.

E-mail: parajubaea@gmail.com

* Corresponding author: gabriele.galasso@comune.milano.it

(C) 2019 Marco Martignoni, Enrico Banfi, Gabriele Galasso
}

Received: 21 December 2018

Accepted for publication: 17 April 2019

Online publication: 24 October 2019
Casorate Sempione, Ferno, Lonate Pozzolo, Samarate, Somma Lombardo e Vizzola Ticino. Le campagne di raccolta si sono svolte da agosto 2010 a settembre 2018 e i campioni sono conservati presso l'erbario del Museo di Storia Naturale di Milano (MSNM). L'elenco floristico, organizzato seguendo l'ordinamento e la nomenclatura delle recenti checklist della flora italiana, annovera 395 taxa di rango specifico e sottospecifico $(9,35 \%$ dell'intera flora lombarda), appartenenti a 247 generi e 72 famiglie. Le entità native sono 318 , quelle alloctone 77 . Il rapporto autoctone/alloctone risulta molto simile a quello calcolato a livello regionale $(4,10$ vs. 4,42$)$. Il contingente endemico annovera due taxa: Centaurea nigrescens subsp. pinnatifida, endemica italiana, ed Euphrasia cisalpina, endemica alpica. L'aeroporto di Malpensa rimane ad oggi l'unico sito confermato a livello nazionale per Lepidium heterophyllum e, a livello regionale, per Festuca muralis ed Euphrasia cisalpina. Le caratteristiche ambientali dell'area di studio e l'analisi dei dati verranno presentati in un secondo contributo.

Parole chiave: aeroporto, Euphrasia cisalpina, Festuca muralis, Lepidium heterophyllum, Malpensa.

\section{INTRODUCTION}

Airport areas include, among their flight infrastructures, large green spaces (Fig. 1c) that, not having production purposes, are managed through minimum disturbance interventions on habitats, with potential positive effects on the conservation of biodiversity (Martignoni et al., 2016).

We hereby present the checklist of the vascular flora of the green areas of Milan Malpensa airport, which includes well preserved semi-natural habitats, like heathlands, grasslands and meadows. These areas are managed through periodic mowing that hinders the ecological succession to the forest climax vegetation. The ecological characteristics of the study area and the data analysis will be discussed in a second contribution.

\section{MATERIALS AND METHODS}

The study area includes the entire airport of Milan Malpensa (Fig.1c) that extends for $12.44 \mathrm{~km}^{2}$ in the south-western portion of the province of Varese (Lombardy, Italy) (Fig. 1a) in the administrative districts of Cardano al Campo, Casorate Sempione, Ferno, Lonate Pozzolo, Samarate, Somma Lombardo and Vizzola Ticino (Fig. 1b). The altitude ranges from 206.0 to 234.6 $\mathrm{m}$ a.s.l. The soil lays on fluvio-glacial gravelly sediments (Servizio Geologico d'Italia, 1967).

Average annual rainfall is $1211.7 \mathrm{~mm}$, well distributed in the vegetative season with two equinoctial maxima 


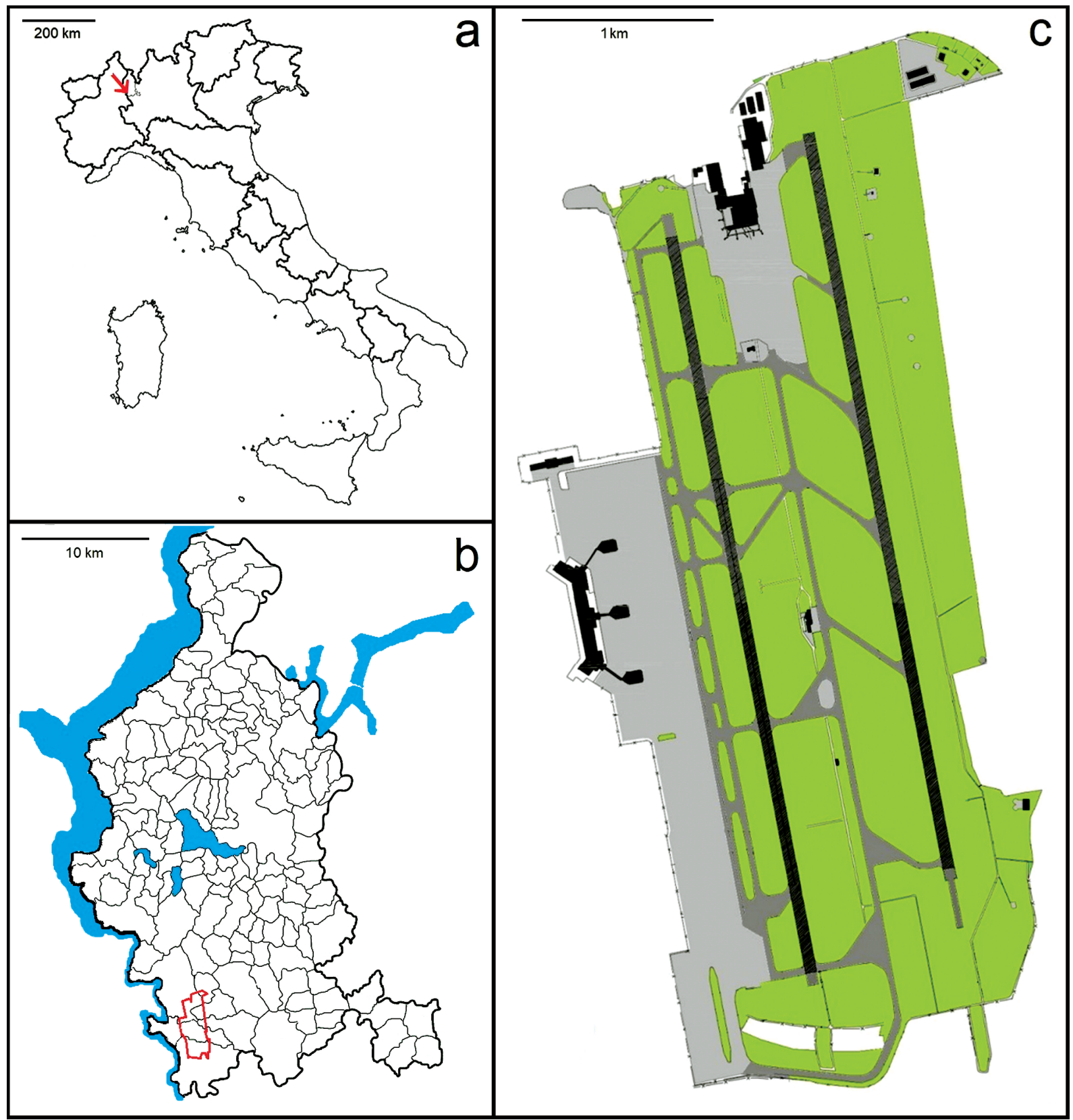

Fig. 1 - Location of the study area at national (a: red arrow) and provincial level (b: red contour) and a simplified map of the Milan Malpensa airport (c): green color highlights the vegetated areas. / Localizzazione dell'area di studio a livello nazionale (a: freccia rossa) e provinciale (b: contorno rosso) e una mappa semplificata del sedime aeroportuale di Milano Malpensa (c): il colore verde evidenzia le aree vegetate. (Drawing / disegno: M. Martignoni).

(May and October) and two minima, one in winter (January and December) the other in summer (July); climatic drought do not occurs. Annual average temperature is $11.6^{\circ} \mathrm{C}$, with the hottest month in summer (July, $\left.22.2^{\circ} \mathrm{C}\right)$ and the coldest in winter (January, $1.7^{\circ} \mathrm{C}$ ) (Centro Nazionale di Meteorologia e Climatologia Aeronautica, 2008). According to Rivas-Martínez et al. (2015), the bioclimate is temperate continental, the thermotype mesotemperate, the ombrotype humid. The potential natural vegetation is a deciduous upland oak forest (pedunculate and/or sessile oakwood) (Società per azioni Esercizi Aeroportuali, 2010).

The collection campaigns were carried out in the period between August 2010 and September 2018. The specimens are kept in the herbarium facility of the Natural History Museum of Milan (MSNM; acronym according to Thiers, 2018). The floristic list was organized following the arrangement and the nomenclature of the recently published Italian vascular flora checklists (Bartolucci et al., 2018; Galasso et al., 2018), except for native hybrids not considered in the above-mentioned checklist.

For each taxon, the following information is reported: accepted name, cryptogenic and alien status in Lombardy. These abbreviations are used in the floristic list: A (archaeophyte), N (neophyte), CAS (casual), NAT (naturalized), INV (invasive), C (cryptogenic, i.e. doubtfully native taxon, whose origin of occurrence in Lombardy is unknown). 


\section{RESULTS}

The spontaneous vascular flora of Malpensa airport amounts to 395 taxa of specific and subspecific rank $(9.35 \%$ of the entire flora of Lombardy: Galasso et al., 2018) belonging to 247 genera and 72 families. The native taxa are 318 including 2 hybrids, while the alien ones are 77 . The native/aliens ratio is comparable to the one calculated at the regional level (4.10 vs. 4.42). According to Bartolucci et al. (2018), these statistics does not take into account the native hybrids. The endemic contingent includes Centaurea nigrescens subsp. pinnatifida, Italian endemic, and Euphrasia cisalpina, endemic to the Alps. The Malpensa airport is still the only site confirmed at the national level for Lepidium heterophyllum (Martignoni et al., 2016) and, at the regional level, for Festuca muralis (Martignoni et al., 2016) and Euphrasia cisalpina (Martignoni, 2014).

\section{CHECKLIST}

Ferns and fern allies

Equisetaceae

Equisetum arvense L.

Dennstaedtiaceae

Pteridium aquilinum (L.) Kuhn subsp. aquilinum

Dryopteridaceae

Dryopteris filix-mas (L.) Schott

Gymnosperms

Pinaceae

N NAT Pinus rigida Mill.

Pinus sylvestris L.

\section{Angiosperms}

Orchidaceae

Anacamptis morio (L.) R.M.Bateman, Pridgeon \& M.W.Chase

Neotinea ustulata (L.) R.M.Bateman, Pridgeon \& M.W.Chase

Platanthera bifolia (L.) Rich.

Asphodelaceae

N NAT Hemerocallis fulva (L.) L.

Amaryllidaceae

Allium vineale $\mathrm{L}$.

Narcissus poëticus L.

A CAS Narcissus pseudonarcissus L. subsp.

pseudonarcissus s.l.

Notes: There are only double-flowered cultivars (Narcissus Group 4), which cannot be traced for sure to a particular species or hybrid; following the suggestion of Banfi \& Galasso (2010), they were included in this species. Note: Sono presenti soltanto cultivar a fiore doppio (Narcissus Gruppo 4), che non possono essere ricondotte con sicurezza a una specie o a un determinato ibrido; analogamente a Banfi \& Galasso (2010) sono state incluse in questa specie.

A CAS Narcissus $\times$ incomparabilis Mill. nothosubsp. incomparabilis $(=N$. poëticus $\mathrm{L} . \times$ N. pseudonarcissus L. subsp. pseudonarcissus)

Asparagaceae

Anthericum ramosum $\mathrm{L}$.

Asparagus officinalis L. subsp. officinalis

Muscari comosum (L.) Mill.

Ornithogalum divergens Boreau

Polygonatum multiflorum (L.) All.

Juncaceae

Juncus articulatus L. subsp. articulatus

Juncus bufonius L.

Juncus effusus L. subsp. effusus

N INV Juncus tenuis Willd.

Luzula campestris (L.) DC. subsp. campestris

Luzula multiflora (Ehrh.) Lej. subsp. multiflora

Cyperaceae

Carex brizoides L.

Carex caryophyllea Latourr.

Carex divulsa Stokes

Carex hirta L.

Carex leporina $\mathrm{L}$.

Carex pallescens $\mathrm{L}$.

Carex spicata Huds.

Cyperus esculentus L.

N INV Cyperus microiria Steud.

Poaceae

Agrostis gigantea Roth subsp. gigantea

Agrostis stolonifera L. subsp. stolonifera

Aira caryophyllea $\mathrm{L}$.

Anisantha diandra (Roth) Tutin ex Tzvelev

Anisantha madritensis (L.) Nevski subsp. madritensis

Anisantha sterilis (L.) Nevski

Anisantha tectorum (L.) Nevski

Anthoxanthum odoratum L.

Arrhenatherum elatius (L.) P.Beauv. ex

J.Presl \& C.Presl subsp. elatius

A NAT Arundo donax L.

Avena barbata Pott ex Link

Avenella flexuosa (L.) Drejer subsp. flexuosa

Avenula pubescens (Huds.) Dumort. subsp. pubescens

Bothriochloa ischaemum (L.) Keng

Brachypodium rupestre (Host) Roem. \& Schult.

Bromopsis erecta (Huds.) Fourr. subsp. erecta

Bromopsis erecta (Huds.) Fourr. subsp. stenophylla (Link) H.Scholz \& Valdés

N INV Bromopsis inermis (Leyss.) Holub subsp. inermis

Bromus hordeaceus L. subsp. hordeaceus

Bromus hordeaceus L. subsp. molliformis (J.Lloyd ex Billot) Maire \& Weiller 
Calamagrostis epigejos (L.) Roth subsp. epigejos

N NAT Ceratochloa cathartica (Vahl) Herter

Cynodon dactylon (L.) Pers.

Dactylis glomerata L. subsp. glomerata

Danthonia decumbens (L.) DC. subsp. decumbens

N NAT Dichanthelium acuminatum (Sw.) Gould \& C.A.Clark subsp. implicatum (Scribn.) Freckmann \& Lelong

C Digitaria sanguinalis (L.) Scop.

Echinochloa crus-galli (L.) P.Beauv. subsp. crus-galli

Elymus repens (L.) Gould subsp. repens

N INV Eragrostis pectinacea (Michx.) Nees

Festuca bromoides L.

Festuca danthonii Asch. \& Graebn. subsp. danthonii

Festuca filiformis Pourr.

Festuca lachenalii (C.C.Gmel.) Spenn.

Festuca muralis Kunth

Festuca myuros L. subsp. myuros

Festuca rubra L. subsp. juncea (Hack.) K.Richt.

Festuca rubra L. subsp. rubra

Festuca stricta Host subsp. trachyphylla (Hack.) Patzke ex Pils

Holcus lanatus L. subsp. lanatus

Holcus mollis L. subsp. mollis

Hordeum murinum L. subsp. leporinum (Link) Arcang.

Koeleria pyramidata (Lam.) P.Beauv.

Lolium arundinaceum (Schreb.) Darbysh. subsp. arundinaceum

Lolium multiflorum Lam.

Lolium perenne $\mathrm{L}$.

Molinia arundinacea Schrank

N INV Muhlenbergia schreberi J.F.Gmel.

N INV Panicum dichotomiflorum Michx.

N NAT Paspalum dilatatum Poir.

Phleum pratense L. subsp. pratense

Phragmites australis (Cav.) Trin. ex Steud. subsp. australis

Poa angustifolia $\mathrm{L}$.

Poa annua L.

Poa bulbosa L. subsp. bulbosa

Poa compressa L.

Poa pratensis L. subsp. pratensis

Poa trivialis L.

N NAT Setaria italica (L.) P.Beauv. subsp. pycnocoma (Steud.) de Wet

C Setaria italica (L.) P.Beauv. subsp. viridis (L.) Thell.

Setaria pumila (Poir.) Roem. \& Schult.

A INV Sorghum halepense (L.) Pers.

N NAT Sporobolus neglectus Nash

N INV Sporobolus vaginiflorus (Torr. ex A.Gray) Alph.Wood

Tragus racemosus (L.) All.

A CAS Triticum aestivum L. subsp. aestivum
Berberidaceae

N NAT Mahonia aquifolium (Pursh) Nutt.

Ranunculaceae

Clematis vitalba $\mathrm{L}$.

Ficaria verna Huds. subsp. verna

Ranunculus acris L. subsp. acris

Ranunculus bulbosus L.

Papaveraceae

Chelidonium majus L.

Fumaria officinalis L. subsp. wirtgenii (W.D.J.Koch) Arcang.

C Papaver rhoeas L. subsp. rhoeas

Crassulaceae

Sedum sexangulare L.

Saxifragaceae

Saxifraga tridactylites L.

Vitaceae

N INV Parthenocissus inserta (A.Kern.) Fritsch

N CAS Parthenocissus tricuspidata (Siebold \& Zucc.) Planch.

N NAT Vitis $\times$ bacoi Ardenghi, Galasso \& Banfi

$\mathrm{N}$ INV Vitis $\times$ koberi Ardenghi, Galasso, Banfi \& Lastrucci

N NAT Vitis $\times$ novae-angliae Fernald

Fabaceae

N CAS Albizia julibrissin Durazz.

Astragalus glycyphyllos L.

Cytisus nigricans L. subsp. nigricans

Cytisus scoparius (L.) Link subsp. scoparius

Ervilia hirsuta (L.) Opiz

A NAT Galega officinalis L.

Genista germanica L.

Genista tinctoria L.

Hippocrepis comosa L. subsp. comosa

Lathyrus sylvestris L. subsp. sylvestris

Lotus corniculatus L. subsp. corniculatus

A NAT Medicago sativa L.

Onobrychis viciifolia Scop.

Ononis spinosa L. subsp. spinosa

N INV Pueraria lobata (Willd.) Ohwi

N INV Robinia pseudoacacia L.

N INV Robinia viscosa Vent.

Securigera varia (L.) Lassen

Trifolium arvense $\mathrm{L}$. subsp. arvense

Trifolium campestre Schreb.

Trifolium pratense $\mathrm{L}$. subsp. pratense

Trifolium repens $\mathrm{L}$.

Trigonella alba (Medik.) Coulot \& Rabaute

Trigonella officinalis (L.) Coulot \& Rabaute

Vicia angustifolia $\mathrm{L}$.

Vicia cracca L.

Vicia villosa Roth

Polygalaceae

Polygala vulgaris L. subsp. vulgaris 
Rosaceae

Agrimonia eupatoria L. subsp. eupatoria

N CAS Chaenomeles speciosa (Sweet) Nakai

Drymocallis rupestris (L.) Soják

Filipendula vulgaris Moench

Fragaria moschata Weston

Fragaria vesca L. subsp. vesca

Potentilla argentea L.

Notes: Some plants could correspond to the hybrid with $P$. intermedia.

Note: Sono presenti alcune piante che potrebbero corrispondere all'ibrido con $P$. intermedia.

Potentilla argentea L. $\times$ Potentilla verna $\mathrm{L}$.

Potentilla erecta (L.) Raeusch.

Potentilla inclinata Vill.

N INV Potentilla indica (Andrews) Th. Wolf

N CAS Potentilla intermedia L.

Potentilla pusilla Host

Potentilla recta L. subsp. recta

Potentilla reptans L.

Poterium sanguisorba L. subsp. sanguisorba

A NAT Prunus cerasifera Ehrh.

A NAT Prunus domestica L.

A CAS Prunus persica (L.) Batsch

N INV Prunus serotina Ehrh.

Prunus spinosa L. subsp. spinosa

Rubus caesius L.

Rubus praecox Bertol.

Rubus sect. Corylifolii Lindl.

N NAT Spiraea salicifolia L.

Rhamnaceae

Frangula alnus Mill. subsp. alnus

Ulmaceae

Ulmus minor Mill. subsp. minor

Cannabaceae

N INV Humulus japonicus Siebold \& Zucc.

Humulus lupulus L.

Moraceae

Ficus carica $\mathrm{L}$.

A NAT Morus alba L.

Urticaceae

Parietaria judaica L.

Urtica dioica L. subsp. dioica

Urtica urens $\mathrm{L}$.

Fagaceae

Castanea sativa Mill.

Quercus robur L. subsp. robur

Quercus rubra L.

Juglandaceae

C Juglans regia $\mathrm{L}$.

Betulaceae

Betula pendula Roth
Carpinus betulus L.

Corylus avellana L.

Celastraceae

Euonymus europaeus L.

Oxalidaceae

N NAT Oxalis articulata Savigny

N INV Oxalis dillenii Jacq.

Violaceae

Viola alba Besser subsp. dehnhardtii (Ten.) W.Becker

Viola arvensis Murray subsp. arvensis

Viola canina L. subsp. canina

Viola odorata L.

Salicaceae

Populus alba L.

Populus canescens (Aiton) Sm.

Populus nigra L. subsp. nigra

Populus tremula L.

Salix alba L.

Salix caprea L.

Salix purpurea L. subsp. purpurea

Hypericaceae

N CAS Hypericum calycinum L.

Hypericum humifusum $\mathrm{L}$.

Hypericum perforatum L. subsp. veronense (Schrank) Ces.

Euphorbiaceae

Euphorbia cyparissias L.

Euphorbia esula L. subsp. esula

Euphorbia helioscopia L. subsp. helioscopia

A NAT Euphorbia lathyris L.

N INV Euphorbia maculata L.

Geraniaceae

Erodium cicutarium (L.) L'Hér.

Geranium columbinum L.

Geranium molle L.

Onagraceae

Chamaenerion dodonaei (Vill.) Schur ex Fuss

N NAT Oenothera biennis L.

N NAT Oenothera chicaginensis de Vries ex Renner

\& Cleland

N INV Oenothera stucchii Soldano

Lythraceae

Lythrum salicaria L.

Sapindaceae

Acer campestre L.

Acer pseudoplatanus L.

Simaroubaceae

N INV Ailanthus altissima (Mill.) Swingle 
Cistaceae

Helianthemum nummularium (L.) Mill. subsp. obscurum (Čelak.) Holub

Malvaceae

Malva alcea L.

Malva moschata L.

Malva sylvestris L.

Resedaceae

Reseda lutea L. subsp. lutea

Brassicaceae

Alliaria petiolata (M.Bieb.) Cavara \& Grande

Arabidopsis thaliana (L.) Heynh.

Barbarea vulgaris $\mathrm{R}$. Br.

A NAT Brassica napus L.

Capsella bursa-pastoris (L.) Medik. subsp. bursa-pastoris

Cardamine hirsuta L.

Diplotaxis tenuifolia (L.) DC.

Draba verna L. s.l.

Notes: The observed individuals are not attributable for sure to any of the subspecies reported by Bartolucci et al. (2018), which we believe are devoid of systematic significance.

Note: Gli individui presenti non sono attribuibili con sicurezza a nessuna delle sottospecie riportate da Bartolucci et al. (2018), che secondo noi sono prive di significato sistematico.

Erucastrum nasturtiifolium (Poir.)

O.E.Schulz subsp. nasturtiifolium

N NAT Lepidium heterophyllum Benth.

$\mathrm{N}$ INV Lepidium virginicum L. subsp. virginicum

Microthlaspi perfoliatum (L.) F.K.Mey.

Raphanus raphanistrum L. subsp. raphanistrum

N NAT Rorippa armoracioides (Tausch) Fuss

N NAT Rorippa austriaca (Crantz) Besser

Rorippa sylvestris (L.) Besser subsp. sylvestris

Teesdalia nudicaulis (L.) R.Br.

Turritis glabra L.

Santalaceae

Thesium linophyllon L.

Polygonaceae

Fallopia convolvulus (L.) Á.Löve

Persicaria lapathifolia (L.) Delarbre subsp. lapathifolia

Persicaria lapathifolia (L.) Delarbre subsp. pallida (With.) Á.Löve

Polygonum arenastrum Boreau

N INV Reynoutria bohemica Chrtek \& Chrtková

Rumex acetosa L. subsp. acetosa

Rumex acetosella L. subsp. acetosella

Rumex acetosella L. subsp. acetoselloides (Balansa) Den Nijs

Rumex obtusifolius L. subsp. obtusifolius

Caryophyllaceae

Arenaria serpyllifolia L. subsp. serpyllifolia
Atocion armeria (L.) Raf.

Atocion rupestre (L.) Oxelman

Cerastium arvense L. subsp. arvense

Cerastium glomeratum Thuill.

Cerastium holosteoides Fr.

Cerastium ligusticum Viv.

Cerastium semidecandrum L.

Dianthus armeria L. subsp. armeria

Petrorhagia prolifera (L.) P.W.Ball \& Heywood

Petrorhagia saxifraga (L.) Link subsp. saxifraga

Psammophiliella muralis (L.) Ikonn.

Sabulina tenuifolia (L.) Rchb. subsp. tenuifolia

Sagina apetala Ard. subsp. apetala

Saponaria officinalis L.

Scleranthus perennis L. subsp. perennis

Scleranthus polycarpos L.

Silene gallica L.

Silene latifolia Poir.

Silene nutans L. subsp. insubrica (Gaudin) Soldano

Silene vulgaris (Moench) Garcke subsp. vulgaris

Spergula arvensis L.

Stellaria media (L.) Vill. subsp. media

Amaranthaceae

N INV Amaranthus hybridus L. subsp. hybridus

Chenopodiaceae

Chenopodium album L. subsp. album

Dysphania botrys (L.) Mosyakin \& Clemants

N NAT Dysphania pumilio (R.Br.) Mosyakin \& Clemants

Phytolaccaceae

N INV Phytolacca americana L.

Portulacaceae

Portulaca nitida (Danin \& H.G.Baker) Ricceri \& Arrigoni

Primulaceae

Lysimachia arvensis (L.) U.Manns \& Anderb. subsp. arvensis

Ericaceae

Calluna vulgaris (L.) Hull

Rubiaceae

Cruciata glabra (L.) C.Bauhin ex Opiz

Cruciata pedemontana (Bellardi) Ehrend.

Galium aparine L.

Galium mollugo L.

Galium parisiense L.

Galium rubrum L.

Galium verum L. subsp. verum

Sherardia arvensis L. 
Gentianaceae

Centaurium erythraea Rafn subsp. erythraea

Apocynaceae

N NAT Asclepias syriaca L.

Vinca minor L.

Vincetoxicum hirundinaria Medik. subsp. hirundinaria

Convolvulaceae

Convolvulus arvensis $\mathrm{L}$.

Convolvulus sepium L.

N INV Cuscuta campestris Yunck.

Solanaceae

Solanum nigrum L.

Boraginaceae

Echium vulgare L. subsp. vulgare

Myosotis ramosissima Rochel subsp. ramosissima

Oleaceae

N CAS Forsythia viridissima Lindl.

Fraxinus excelsior L. subsp. excelsior

Fraxinus ornus L. subsp. ornus

N INV Ligustrum sinense Lour.

Plantaginaceae

Anarrhinum bellidifolium (L.) Willd.

Linaria angustissima (Loisel.) Borbás

Linaria pelisseriana (L.) Mill.

Plantago lanceolata L.

Plantago major L.

Veronica arvensis L.

N INV Veronica filiformis Sm.

Veronica hederifolia L.

Veronica officinalis L.

Scrophulariaceae

N INV Buddleja davidii Franch.

Scrophularia canina L.

Scrophularia nodosa L.

Verbascum densiflorum Bertol.

Verbascum lychnitis L.

Verbascum nigrum L.

Verbascum phlomoides L.

Lamiaceae

Ajuga genevensis L.

Ajuga reptans L.

Betonica officinalis L.

Clinopodium nepeta (L.) Kuntze subsp. nepeta

Clinopodium vulgare L. subsp. vulgare

Glechoma hederacea L.

Lamium maculatum L.

Lamium purpureum $\mathrm{L}$.

Mentha $\times$ piperita L. nothosubsp. piperita $(=M$. aquatica L. subsp. aquatica $\times M$. spicata L.)
N INV

Mentha spicata L.

Prunella laciniata (L.) L.

Prunella vulgaris L. subsp. vulgaris

Salvia pratensis L. subsp. pratensis

Teucrium scorodonia L.

Thymus oenipontanus Heinr.Braun ex Borbás Ziziphora acinos (L.) Melnikov subsp. acinos

Orobanchaceae

Euphrasia cisalpina Pugsley

Verbenaceae

Verbena officinalis L.

Campanulaceae

Campanula rapunculoides L. subsp. rapunculoides

Campanula rapunculus L.

Asteraceae

Achillea millefolium L. s.1.

Notes: Populations are difficult to ascribe; according to Valentino Casolo (in litt.), they would partially correspond to A. collina (Becker ex Wirtg.) Heimerl, A. millefolium subsp. millefolium, A. collina $\times$

A. millefolium, and A. collina $\times$ A. setacea.

Note: Le popolazioni sono difficilmente attribuibili; secondo Valentino Casolo (in litt.) sarebbero parzialmente corrispondenti ad $A$. collina (Becker ex Wirtg.) Heimerl, A. millefolium subsp. millefolium, A. collina $\times$ A. millefolium e A. collina $\times$ A. setacea .

Achillea setacea Waldst. \& Kit.

Notes: According to Valentino Casolo (in litt.), some plants could correspond to hybrids with A. roseoalba Ehrend. and with A. collina (Becker ex Wirtg.) Heimerl. Note: Secondo Valentino Casolo (in litt.) alcune piante potrebbero corrispondere a ibridi con $A$. roseoalba Ehrend. e con A. collina (Becker ex Wirtg.) Heimerl.

Ambrosia artemisiifolia L.

Anthemis arvensis L. subsp. arvensis Arctium minus (Hill) Bernh.

N INV Artemisia annua L.

N INV Artemisia verlotiorum Lamotte

Artemisia vulgaris L.

Bellis perennis L.

$\mathrm{N}$ INV Bidens frondosa $\mathrm{L}$.

Carduus nutans L. subsp. leiophyllus (Petrovič) Stoj. \& Stef.

Centaurea deusta Ten.

Centaurea jacea L. subsp. gaudinii (Boiss. \& Reut.) Gremli

Centaurea nigrescens Willd. subsp. pinnatifida (Fiori) Dostál

Chondrilla juncea L.

Cichorium intybus L.

Cirsium arvense (L.) Scop.

Cirsium vulgare (Savi) Ten. subsp. vulgare

Crepis capillaris (L.) Wallr.

Crepis foetida L. subsp. foetida

Crepis setosa Haller f.

N INV Erigeron annuus (L.) Desf.

N INV Erigeron canadensis L.

Eupatorium cannabinum L. subsp. cannabinum 
N INV Helianthus tuberosus L.

Hieracium umbellatum L. subsp. umbellatum Hypochaeris maculata $\mathrm{L}$.

Hypochaeris radicata $\mathrm{L}$.

Lactuca sativa L. subsp. serriola (L.) Galasso, Banfi, Bartolucci \& Ardenghi

Leontodon hispidus L. subsp. hispidus

Leucanthemum ircutianum DC. subsp. ircutianum

Logfia minima (Sm.) Dumort.

Matricaria chamomilla L.

Picris hieracioides L. subsp. hieracioides

Pilosella officinarum Vaill.

Pilosella piloselloides (Vill.) Soják subsp. piloselloides

Pilosella velutina (Hegetschw.) F.W.Schultz \& Sch.Bip.

Scorzoneroides autumnalis (L.) Moench

N INV Senecio inaequidens DC.

Senecio vulgaris L. subsp. vulgaris

N INV Solidago gigantea Aiton

Sonchus asper (L.) Hill subsp. asper

C Sonchus tenerrimus L.

N INV Symphyotrichum lanceolatum (Willd.)

G.L.Nesom

N NAT Symphyotrichum novi-belgii (L.) G.L.Nesom

Taraxacum F.H.Wigg. sect. Taraxacum

Tragopogon dubius Scop.

Viburnaceae

Sambucus nigra L.

Caprifoliaceae

N INV Lonicera japonica Thunb.

Dipsacaceae

Knautia collina Jord.

Knautia transalpina (Christ ex Gremli) Dalla Torre

Notes: The name was attributed to these authors according to the checked protologues.

Note: L'indicazione degli autori del nome è stata fatta verificando i protologhi.

Scabiosa columbaria L. subsp. portae

(A.Kern. ex Huter) Hayek

Scabiosa triandra L.

Valerianaceae

Valerianella locusta (L.) Laterr.

Araliaceae

Hedera helix L. subsp. helix

Apiaceae

Daucus carota L. subsp. carota

Oreoselinum nigrum Delarbre

\section{Acknowledgments}

We thank Società per azioni Esercizi Aeroportuali (S.E.A. S.p.A.), for authorizing the collection of specimens and the publication of this contribution, and in par- ticular Davide Pisoni and Elena Portone. For the identification of the critical groups are indebted to Nicola M.G. Ardenghi (Festuca, Vitis), Fabrizio Bartolucci (Thymus), Valentino Casolo (Achillea), Günter Gottschlich (Pilosella), Thomas Gregor (Potentilla p.p.), and Adriano Soldano (Oenothera). We are also grateful to Enzo Meneguzzo for reporting Anisantha diandra that he noticed from a shuttle bus while boarding a plane.

\section{REFERENCES}

Banfi E. \& Galasso G. (eds.), 2010 - La flora esotica lombarda. Museo di Storia Naturale di Milano, Milano. (+ Cd-rom).

Bartolucci F., Peruzzi L., Galasso G., Albano A., Alessandrini A., Ardenghi N.M.G., Astuti G., Bacchetta G., Ballelli S., Banfi E., Barberis G., Bernardo L., Bouvet D., Bovio M., Cecchi L., Di Pietro R., Domina G., Fascetti S., Fenu G., Festi F., Foggi B., Gallo L., Gottschlich G., Gubellini L., Iamonico D., Iberite M., Jiménez-Mejías P., Lattanzi E., Marchetti D., Martinetto E., Masin R.R., Medagli P., Passalacqua N.G., Peccenini S., Pennesi R., Pierini B., Poldini L., Prosser F., Raimondo F.M., RomaMarzio F., Rosati L., Santangelo A., Scoppola A., Scortegagna S., Selvaggi A., Selvi F., Soldano A., Stinca A., Wagensommer R.P., Wilhalm T. \& Conti F., 2018 - An updated checklist of the vascular flora native to Italy. Plant Biosystems, 152 (2): 179-303. < doi: 10.1080/11263504.2017.1419996>

Centro Nazionale di Meteorologia e Climatologia Aeronautica, 2008 Atlante climatico d'Italia: 1971-2000. Available from: $<$ http://clima. meteoam.it/AtlanteClimatico/pdf/(066)Milano\%20Malpensa.pdf> (retrieved on 13 March 2019).

Galasso G., Conti F., Peruzzi L., Ardenghi N.M.G., Banfi E., CelestiGrapow L., Albano A., Alessandrini A., Bacchetta G., Ballelli S., Bandini Mazzanti M., Barberis G., Bernardo L., Blasi C., Bouvet D., Bovio M., Cecchi L., Del Guacchio E., Domina G., Fascetti S., Gallo L., Gubellini L., Guiggi A., Iamonico D., Iberite M., Jiménez-Mejías P., Lattanzi E., Marchetti D., Martinetto E., Masin R.R., Medagli P., Passalacqua N.G., Peccenini S., Pennesi R., Pierini B., Podda L., Poldini L., Prosser F., Raimondo F.M., RomaMarzio F., Rosati L., Santangelo A., Scoppola A., Scortegagna S., Selvaggi A., Selvi F., Soldano A., Stinca A., Wagensommer R.P., Wilhalm T. \& Bartolucci F., 2018 - An updated checklist of the vascular flora alien to Italy. Plant Biosystems, 152 (3): 556-592. <doi: 10.1080/11263504.2018.1441197>

Martignoni M., 2014 - Euphrasia cisalpina Pugsley (Orobanchaceae) nella Brughiera di Gallarate (Lombardia, Italia): dati storici e conferma della stazione nelle aree verdi dell'Aeroporto di Milano Malpensa. Natural History Sciences, 1 (1): 19-24. < doi: 10.4081/ nhs.2014.63>

Martignoni M., Banfi E. \& Galasso G., 2016 - Conservazione della flora in aree ad alta urbanizzazione: il caso dell'aeroporto di Milano Malpensa. In: Minilavori della Riunione scientifica del Gruppo per la Floristica, Sistematica ed Evoluzione. 21-22 ottobre 2016, Roma. Domina G. \& Peruzzi L. (eds.). Notiziario della Società Botanica Italiana, 0: 55-56.

Rivas-Martínez S., Rivas-Sáenz S., Penas A. \& Díaz T.E., 2015 Computerized bioclimatic maps of the World: bioclimates of Europe and Mideast. Centro de Investigaciones Fitosociólogicas, Madrid. Available from: <http://www.globalbioclimatics.org/form/ maps.htm> (retrieved on 13 March 2019).

Servizio Geologico d'Italia, 1967 - Carta Geologica d'Italia alla scala 1:100.000, F. 44 Novara (II edizione).

Società per azioni Esercizi Aeroportuali (S.E.A. S.p.A.), 2010 Studio di Impatto Ambientale. Nuovo Master Plan Aeroportuale dell'Aeroporto di Malpensa. Segrate (Milano).

Thiers B., 2018 onwards - Index Herbariorum: A global directory of public herbaria and associated staff. Available from: $<\mathrm{http}: / /$ sweetgum.nybg.org/ih/> (retrieved on 1 December 2018). 